\title{
Communautés locales et gibiers dans la région de Daraina, extrême Nord-Est de Madagascar
}

\author{
Hery A. Rakotondravony
}

\begin{abstract}
Wild meats take part in the daily protein sources for many Malagasy rural communities. Many animal species are affected by hunting, which is practiced during almost all seasons in Madagascar. In the Daraina region, animal groups hunted by local people are mainly mammals, birds, fishes, and reptiles. As the natural habitats within this region are actually highly perturbated by human activities, and as this region shows a high population growth, hunting may constitute a severe threat for many species. Sustainable management of these later depends on local population awareness regarding the threats of intensive hunting on animals as well as on the natural ecosystems.
\end{abstract}

\section{INTRODUCTION}

Presque partout en milieu rural à Madagascar, les sources de protéines journalières sont généralement limitées, et sont surtout d'origines végétales. Selon la qualité du sol, le régime climatique et la tradition, ces dernières varient d'une région à une autre, à partir du riz-base de l'alimentation des malgaches-jusqu'à divers rudiments alimentaires (ex. beignets dont la base varie également d'une région à une autre). Pour la majeure partie des malgaches, les protéines animales sont actuellement difficilement accessibles. Signes de richesses et la plupart du temps vendues à des prix hors de la portée des communautés rurales, les viandes de zébus et celles d'autres ovins ne se consomment généralement que pendant les périodes festives.

Pour ces différentes raisons, les viandes sauvages tiennent des places plus ou moins importantes dans I'alimentation des malgaches. Les espèces concernées ainsi que leurs quantités varient d'une région à une autre. Pour les régions marécageuses, fluviatiles ou littorales, les animaux chassés incluent les poissons, les oiseaux, les tortues d'eau douce ou marines, etc. Dans les régions forestières il s'agit d'autres groupes d'animaux tels que les oiseaux, les lémuriens, les sangliers et dans certains cas des reptiles (voir Goodman and Benstead 2003) et les ressources cynégétiques forestières peuvent constituer jusqu'à $27-28 \%$ des produits extraites dans les forêts ( $60 \%$ environ pour les bois de construction; Rabesahala Horning 2003). Le but de ce document est de donner une liste d'animaux sauvages potentiellement chassés dans la région de Daraina et de donner de brefs aperçus sur les conséquences éventuelles des chasses sur leurs populations.
Département de Biologie Animale, Faculté des Sciences, Université d'Antananarivo, BP 906 et Ecology Training Program, WWF, BP 738 Antsakaviro, Antananarivo (101).

E-mail: etp@wwf.mg

\section{LA RÉGION DE DARAINA}

La région de Daraina est comprise entre les fleuves Loky au Sud et Manambato au Nord, dans l'extrême Nord-Est de Madagascar. Le relief, I'existence des reliques forestières et du lac Sahaka (à l'est de la région) conduisent à l'existence de différents types d'habitats où se trouvent une diversité biologique importante. Jusqu'à une période très récente, aucun des habitats forestiers de la région n'était pas protégé et leurs exploitations s'étaient effectuées de manières traditionnelles: cultures sur brûlis, collectes de bois durs et exploitations aurifères artisanales, chasses et pêches.

Sur le littoral est de Daraina se trouve la Réserve de Chasse du lac Sahaka (120 ha) qui a été établie le 13 février 1969 par I'arrêté 0711 MAAER/FIN. Son but était de protéger la faune tributaire de ce lac. La chasse est en principe interdite dans cette réserve; mais au-delà de laquelle, dans la zone appelée Réserve Cynégétique (730 ha), les chasses sont permises, selon I'arrêté ci-dessus, de mai en septembre. Les chasseurs devraient obtenir des permis de collectes délivrés par le Service des Eaux et Forêts de Vohémar, d'Antalaha ou d'Antsiranana, puis les présenter auprès des chefs d'administrations locales et y payer les droits de chasse (Safford 2000).

La population de la région de Daraina est actuellement composée de 30,000 personnes environ. A part les flux migratoires importants des années 80 et 90 relatifs à la découverte de gisements aurifères dans la région, la croissance démographique actuelle reste stationnaire mais importante, environ 4 à $5 \%$ par an entre 1998 et 2003 selon les données de bases de l'Institut National de la Statistique de Madagascar. Ces populations sont d'origines différentes, mais les ethnies présentes dans la région (par ordre d'importance en nombre) sont les Sakalava Anjoaty, les Tsimihety, les Betsimisaraka, les Sakalava Makoa, les Merina et les Antemoro. Ces ethnies possèdent leurs propres traditions (i.e. tabou) à l'égard de la consommation d'animaux sauvages. II existe, néanmoins, des espèces dont la consommation n'est pas en général considérée comme tabou, et sont chassées d'une manière plus ou moins importante.

\section{ESPÈCES ANIMALES CONCERNÉES PAR LA CHASSE ET LA PÊCHE DANS LA RÉGION DE DARAINA}

Nombreuses espèces animales sont chassées par les communautés locales dans la région de Daraina. La chasse à certaines espèces discutées ici (surtout les oiseaux aquatiques) n'était 
pas observée dans la région; et leur inclusion dans cette discussion est essentiellement basée sur le fait qu'elles sont chassées dans d'autres régions de Madagascar. Mais à l'égard de la différence des us et coutumes observée presque partout dans l'île, on ne peut pas fermement rapporter ici qu'ils sont ou ne sont pas activement chassés dans la région.

MAMMIFÈRES Dans l'ensemble la région de Daraina,

I'espèce la plus prisée en tant que gibier est le tenrec (Tenrec ecaudatus, Tenrecidae; figure 1). Cette espèce abonde aussi bien dans les savanes boisées qu'en milieux forestiers. Les populations locales chassent tous les individus qu'ils jugent (de par leurs tailles) subadultes ou adultes, mâles ou femelles, excepté les femelles gravides et les juvéniles. La chasse commence dès la sortie de l'espèce de l'hibernation, à partir de la mi-novembre. Malgré cela, les chasses intensives semblent actuellement ne pas avoir affecter la population de cette espèce dans cette région.

Les Mégachiroptères (fanihy en malgache) sont parmi les animaux fortement chassés à Madagascar (Mackinnon et al 2003). L'espèce Pteropus rufus (Pteropodidae) est représentée par une population importante dans la forêt d'Analabe-Sahaka, sur le littoral est de la région (Safford 2000; obs. pers.). Safford (2000) a estimé cette population entre 1000 à 1500 individus. Les gens du village d'Añaborano, un des villages les plus proches de la lisière de cette forêt, en majorité Sakalava, affirment ne pas consommer de fanihy. Néanmoins, des immigrants observant différentes traditions pourraient constituer une menace pour cette espèce dans la région. En plus, Safford (2000) suspecte des cas probables de consommations de cette espèce du côté d'Ankalotany; et certains hôtels du Nord et du Nord-Ouest de Madagascar mettent généralement les fanihy parmi leurs menus. Par conséquent, la population de Pteropus rufus d'AnalabeSahaka pourrait être concernée par la chasse bien qu'elle ne semble pas être gravement menacée dans les temps actuels.

Le sanglier Potamocherus larvatus (Suidae) est le gibier le plus important en terme de masse corporelle dans cette région et semble également être abondante. Les jeunes gens chassent souvent cette espèce pour la raison qu'elle pourrait engendrer des dégâts importants aux cultures (saisonnières) de riz. Aucune période n'est pas spécialement consacrée pour traquer «l'espèce nuisible». La majeure partie des locaux de la région n'en mange pas la viande; et dans le cas où un individu serait capturé, la viande est souvent donnée aux chiens, quelquefois vendue sur le marché local à bas prix (environ la moitié de celui de la viande de zébus). Les gens qui les consomment appartiennent généralement à d'autres ethnies (surtout les Betsimisaraka et les Merina). L'importance apparente de la taille de la population de cette espèce dans la région de Daraina suggère qu'elle ne semble pas être affectée par la chasse actuellement.

Randrianarisoa et al. (1999) ont fait mentionner quatre espèces de lémuriens qui sont chassées dans la région de Daraina: Eulemur coronatus, E. fulvus sanfordi (Lemuridae); Lepilemur sp. (Megaladapidae) et Propithecus tattersalli (Indridae). La consommation de cette dernière est tabou pour les Sakalava. Cependant, cela ne la met pas totalement à I'abri puisque, par exemple selon M. Théodore (chef quartier d'Ambatoharanana, comm. pers.), dans les années 1990 pendant lesquelles les exploitations aurifères étaient les plus importantes dans la région de Daraina, presque toutes les espèces de lémuriens ont été chassées. Par ailleurs, ce groupe

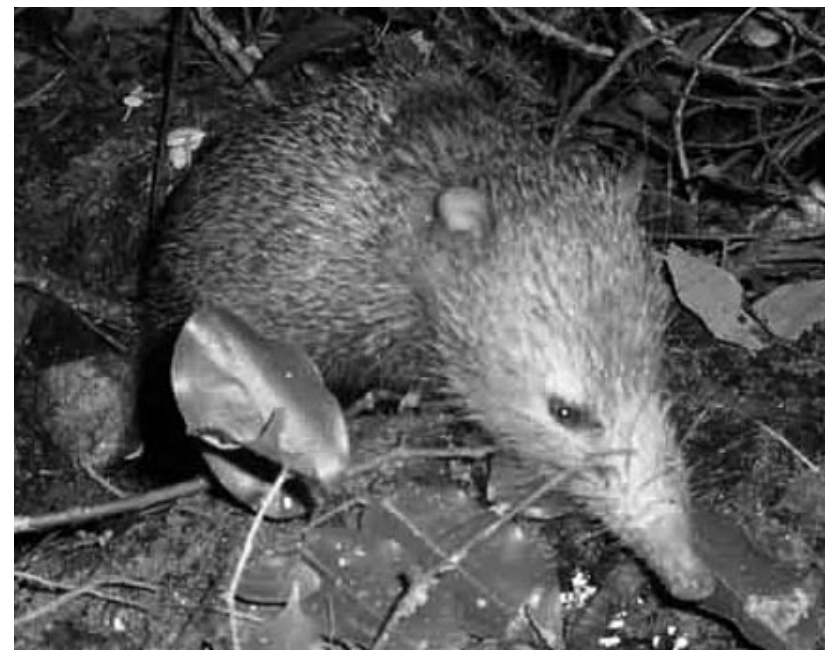

FIGURE 1. Tenrec ecaudatus (Tenrecidae), une des espèces la plus chassée dans la région de Daraina.

est largement chassé presque dans l'ensemble de la région Nord de Madagascar (voir Goodman and Benstead 2003). Dans quelques massifs forestiers, Propithecus tattersalli (Indridae) et Lepilemur spp. (Megaladapidae), se familiarisent facilement à la présence humaine (obs. pers.). Ce type de comportement constituerait un risque important pour ces espèces vis-à-vis de la chasse; mais pourrait également signaler que cette pratique serait plus ou moins limitée dans cette région. En outre, compte tenu de la régression de la couverture forestière dans la région, cette pratique aggraverait la situation de ce groupe d'animal par rapport à sa conservation.

OISEAUX Les tabous et restrictions alimentaires à l'égard

de la faune aviaire semblent moins vigoureux à Madagascar; et les malgaches mangent nombreuses espèces d'oiseaux de différentes tailles (cf. Ekstrom 2003, Goodman and Wilmé 2003, Young 2003). Presque toutes les espèces sont susceptibles d'être chassées, exceptées (en général) les Falconiformes, les Strigiformes et les martins-pêcheurs (Alcedinidae). La région de Daraina n'échappe pas à cette situation. En milieu forestier, I'espèce la plus chassée est Lophotibis cristata (Threskiornithidae), apparemment en raison de sa taille plus importante. Cette espèce a été observée assez fréquemment dans les forêts de Daraina. Les informateurs locaux du côté d'Ankijabe affirment avoir chassé cette espèce pour de nombreuses fois. En dehors de cette espèce en milieu forestier, les autres ne sont chassées vraisemblablement que d'une façon très occasionnelle. Mais dans la région du lac Sahaka, nombreuses espèces d'oiseaux aquatiques existent et sont également susceptibles d'être chassées : Phalarocorax africanus (Phalacrocoracidae); Anhinga rufa (Anhingidae); nombreuses Ardeidae (Ardea sp., Bubulcus sp., Egretta sp., etc.) et Rallidae (ex. Anas sp.).

REPTILES Quatre espèces sont connues consommées par

les gens de la région de Daraina: Acrantophis madagascariensis (Boidae); Pelusios castanoides (Pelomedusidae) et Eretmochelys imbricata et Chelonia mydas (Cheloniidae) (Safford 2000; obs. pers.). Dans la région de Daraina, le boa terrestre A. madagascariensis a été surtout observé dans les savanes boisées ou les forêts dégradées. Les ethnies originaires des zones côtières de Madagascar (Sakalava, Tsimihety, Betsimisaraka et Antemoro) ne consomment pas cette espèce. Actuellement, la chasse semble encore ne pas affecter sa population dans la 
région. La raison serait que les Merina, seuls consommateurs de cette espèce dans la région, semblent les chasser de manière très occasionnelle. C. mydas et E. imbricata, des tortues marines, ont été rapportées nidifiant mais fortement chassées dans certains endroits du littoral est de Daraina (Safford 2000). Concernant $P$. castanoides, bien qu'aucune chasse n'est enregistrée dans la région de Daraina (Safford 2000), cette espèce est consommée dans la région d'Analamerana, environ à $65 \mathrm{~km}$ au nord de la région de Daraina (H.A. Rakotondravony non-publiées). Elle figure par conséquent parmi les espèces qui peuvent potentiellement être des gibiers. Pour Crocodylus niloticus (Crocodylidae), cette espèce n'est pas généralement consommée dans le Nord de Madagascar, et aucune chasse à cette espèce n'était observée ni rapportée dans la région de Daraina.

POISSONS Safford (2000) a signalé sept espèces de poissons récoltés par les pêcheurs dans le lac Sahaka en août 1999: deux espèces allogènes du genre Oreochromis (Cichlidae); et cinq espèces indigènes: Eleotris $s p$. (Eleotridae); Glossogobius sp. (Gobiidae); Mugilidae sp.; Anguilla sp. (Anguillidae) et Megalops cyprinoides (Megalopidae). Cet auteur a fait rapporter également que ces Cichlidae (non-endémiques malgaches) représentaient $90 \%$ de ces récoltes. Lors de nos séjours (février 2004) dans la région du lac Sahaka, ces Cichlidae composaient apparemment $100 \%$ des poissons attrapés par les pêcheurs. La pêche constituait l'activité la plus importante aux alentours du lac bien que cette période était loin de celles autorisées pour pêcher dans la région de Sahaka. Cette activité hors des saisons propices semble affecter les structures des communautés de poissons dans ce lac où les biomasses des espèces endémiques sont déjà largement dominées par les espèces allogènes.

AUTRE TYPE DE CHASSE ET SON IMPACT La collecte de miel (produit par Apis spp., Apidae) constitue une des activités forestières les plus importantes pour les populations de la région de Daraina, surtout pour celles vivant près des lisières forestières. Les miels sont généralement collectés de façon aléatoire, i.e. les collecteurs ignorent si les miels sont prêts ou non à être récoltés; et ils n'hésitent pas d'abattre de gros arbres pour en collecter 1,5 à 3 litres de miel (si la récolte est bonne). Quelques cas d'abattages de gros arbres pour les miels étaient observés dans la région, notamment dans les forêts de Binara, de Bobankora, d'Ampondrabe, d'Andranotsimaty et d'Ambilondambo.

\section{CONCLUSION}

Comme presque partout dans les régions rurales de Madagascar, les animaux sauvages constituent la principale source de protéines animales dans la région de Daraina. Le prélèvement des produits animaux peut affecter aussi bien des espèces particulières que l'ensemble de l'habitat où les produits sont récoltés. Toutefois, sans l'évaluation de la démographie des espèces concernées, il serait encore actuellement difficile de confirmer si les prélèvements affectent ou non ces espèces. Cependant, la croissance démographique importante dans la région suggère des pressions anthropiques de plus en plus importantes. La seule façon de gérer de manière durable ces potentialités naturelles réside dans la sensibilisation des communautés locales faces aux menaces que constituent la pratique intensive de la chasse aussi bien sur les animaux que sur l'ensemble des écosystèmes naturels, et dans la définition et l'application de législations forestières bien définies pour les écosystèmes de la région de Daraina.

\section{REMERCIEMENT}

Je remercie S.M. Goodman pour ses commentaires sur la version préliminaire de ce document.

\section{RÉFÉRENCES}

Ekstrom, J.-M. 2003. Psittaciformes: Coracopsis spp., parrots. In: The Natural History of Madagascar, S.M. Goodman and J.P. Benstead (eds.), pp 1098-1102. The University of Chicago Press, Chicago.

Goodman, S.M., Benstead, J.P. (eds.), 2003. The Natural History of Madagascar. The University of Chicago Press, Chicago.

Goodman, S.M., and Wilmé, L. 2003. Cuculiformes: Coua spp, couas. In: The Natural History of Madagascar, S.M. Goodman and J.P. Benstead (eds.), pp 1102-1108. The University of Chicago Press, Chicago.

Mackinnon, J.L., Hawkins, C.E., and Racey, P.A. 2003. Pteropodidae, fruit bats, fanihy, angavo. In: The Natural History of Madagascar, S.M. Goodman and J.P. Benstead (eds), pp 1299-1302. The University of Chicago Press, Chicago.

Rabesahala Horning, N. 2003. How rules affect conservation outcomes. In: The Natural History of Madagascar, S.M. Goodman and J.P. Benstead (eds.), pp 146-153. The University of Chicago Press, Chicago.

Randrianarisoa, P.M., Rasamison, A.A., and Rakotozafy, L. 1999. Les lémuriens de la région de Daraina: forêt d'Analamazava, forêt de Bekaraoka et forêt de Sahaka. Lemur News 4: 19-21

Safford, R.J. (ed.). 2000. Etude Environnementale et Ecologique du Lac Sahaka, Madagascar. Royal Holloway Institute for Environmental Research, University of London, United Kingdom.

Young, H.G. 2003. Freswater birds. In: The Natural History of Madagascar, S.M. Goodman and J.P. Benstead (eds.), pp 1071-1077. The University of Chicago Press, Chicago. 